\title{
The construction of online course based on Python Language in cloud computing environment
}

\author{
Haiyue Li jianjun Zhang \\ School of Continuing Education \\ Beijing Institute of Technology Beijing China \\ School of Continuing Education \\ Beijing Institute of Technology Beijing China \\ 863712662@qq.com, jjzhang@bit.edu.cn
}

Keywords: cloud computing; Python language; Network Course Construction

\begin{abstract}
Today's society is a decade where new thing and new ideas were popular. With the suggestion of "Internet +", the education is also gradually catch up with the pace of the times, it thrives in the network-based cloud computing environment. Based on the "Internet + " and the cloud computing environment, the research strives to create a network course based on the cloud computing platforms and Python language, so that learners can be self-learning through online courses.
\end{abstract}

\section{Introduction}

In the 2015 "Internet +" first year, the Internet represents a new economic form. The cloud computing of network environment mainly integrate a plurality of relatively low-cost computing into a entity with powerful computing capabilities perfect system through a network. Then by means of software as a service business model, it distributed advanced computing power to the end users.[1] In this study, the system we use is based on the Google cloud computing technology; it runs entirely on the basis of Google web application framework. We should discover the power of Google web applications, and then build programs based on this initial network.

\section{Theoretical and technical support}

The formation and development of online course are inseparable with the development of hardware and software.[3]Any application has certain theory; network curriculum framework also has theoretical support. It includes relevance theory, behavior theory, cognitive theory, situated cognition theory, distributed cognition, information processing theory and humanistic theory. This is the basis.

In order to realize effective integration and a wide range of sharing education resource, Google cloud computing environment learning community system adopts to B/S structure mode, mainly made by the Google cloud computing server and the user of the client. Google cloud computing server, including the two aspects: underlying cloud infrastructure and cloud computing application platform, mainly composed by load balancers, Web servers, application servers and data storage servers. Learning community clients mainly consists of the installed Web browser, a personal computer and other components, you can do all kinds of activities in the learning community, such as participation in study and discussion and so on.

Google Application Engine software applications are available for JAVA or Python programming language, this study we adopt to the Python language. Using App Engine Python Software Develop Kit (SDK) develop and upload Python applications for the Google App Engine software. The Python SDK includes a web server application simulation software engine environment, including local editions and other data storage area, tools contained can be used to test the application, upload application files, manage data store indexes, download log data, and upload 
data to the data store.[5] This paper studies curriculum built can directly call the Python App Engine application to run the server.

\section{Overall software design of the network course}

Network Course contains public information and teaching resources management, learners' cooperation, learner personal space and learner management system, the various subsystems also contains a number of functional modules. Public information and teaching resources management subsystem contains courses, online courseware, teaching resources and software tutorials. Online courses achieves it's making by the Google Enterprise Suite, learners exchange platform is made by Google Sites functional modules. Learner management subsystem includes management and learner registration login information management function module, this subsystem achieve the management of users by integrating Google account system.

The system is a web application running on Google's basic framework, while it integrates Google's rich network applications. As can be seen from the figure3, Google is the basic framework of the system, Google Web applications and their own applications running on this basis. Google Application software Engine provides the basic framework for accessing Google interface, through this interface, you can write a variety of applications based on this basic framework. The developed system will eventually be integrated into our community learning system, and it display with a unified, user-friendly interface to the end user. It's shown in Fig. 1.

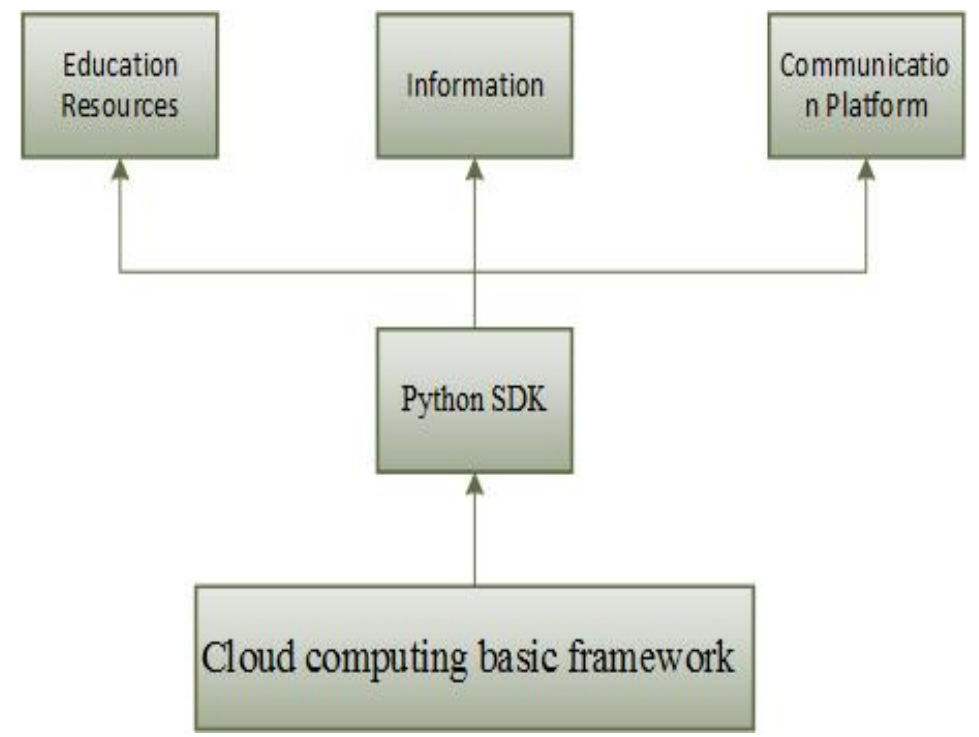

Fig. 1

\section{Build a preliminary network of courses}

There is no need to insist on a pattern with regard to build online courses. Network curriculum emphasizes student-centered. We should take the needs of students into account, and having feedback of problems, to strengthen the interaction. Like the Fig.2, building the network course have to select the contents of the course firstly, then it's necessary to finish the content of teaching instructional design, the overall structure of the planning, making preparation courses required for the production of materials, they can be carried out Courses after everything is ready. 


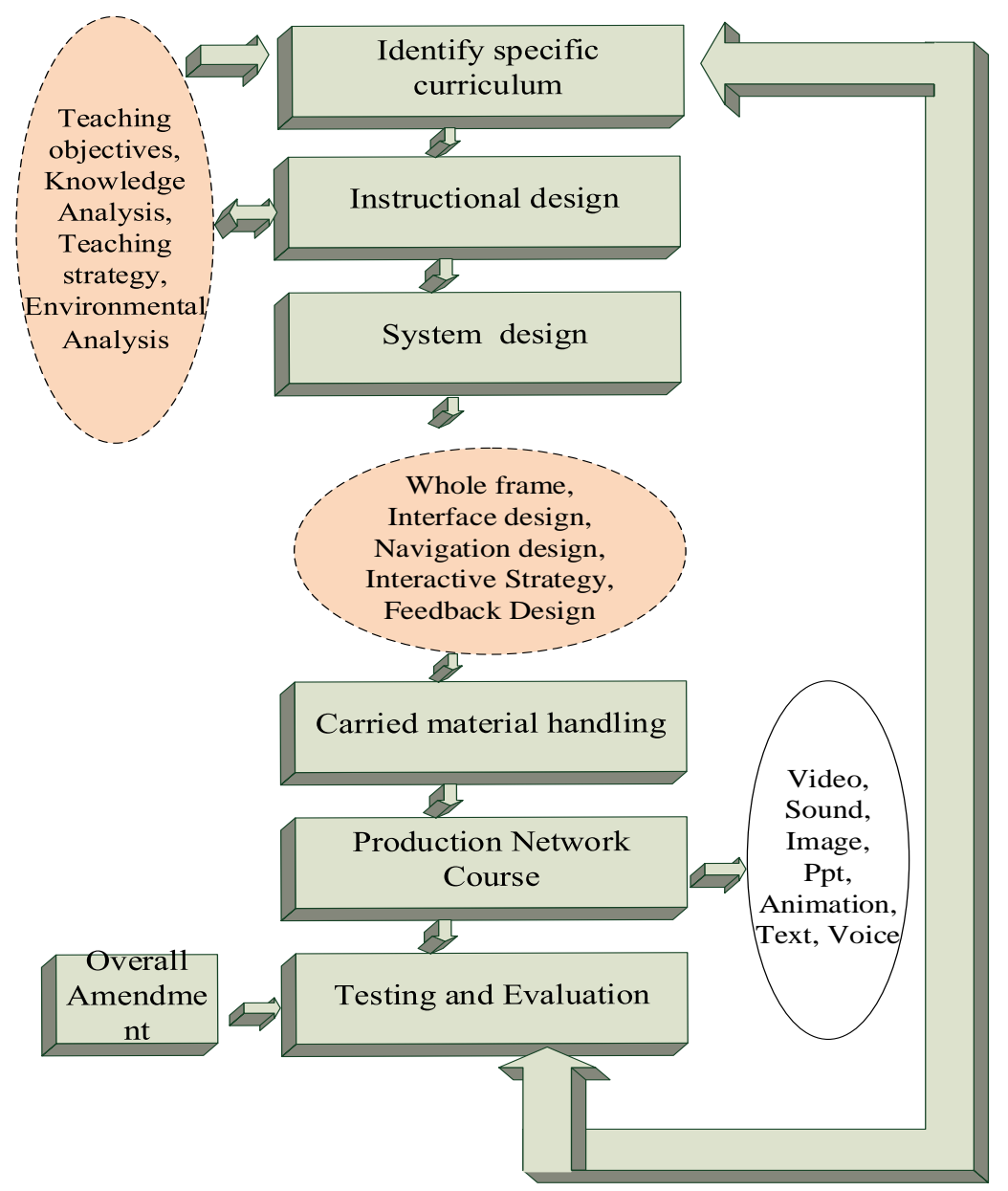

Fig. 2

\section{Overall technical framework}

The online course construction under the cloud computing environment is based on the virtual computing resources and storage space what the massive cloud platform provides. It's a developed learning organization guided by the constructivism theory under the background of the concept of lifelong learning society.

After the supporting of hardware and software architecture design, technical layer contains cloud computing and Python's design, after a huge amount of resources processing and data storage, for production of the course, the main part is the production stage needs analysis, goal setting, determining content. The next stage is the platform to display, mainly including basic information, learning resources and communication platform, tests and examinations, course of study, the results of feedback. Students can achieve online learning courses through pad, mobile terminals, and network computers. We provide a detailed explanation based on the above analysis, the system architecture is shown in Fig.3.

A:According to the learning objectives and requirements of users, directly from the internet database as well as major cloud storage platforms to share information, and the open teaching resources of the Education network, then we automated screening process to integrate a complete set of courses and various learning resources program. The whole uses Python SDK that can achieve.

B:Use Python SDK to achieve personal information management system, including personally identifiable information management and courses.

C:Use Python SDK to achieve the online video player, online courseware browsing, answering system and the collaborative learning system by using Google platform. 


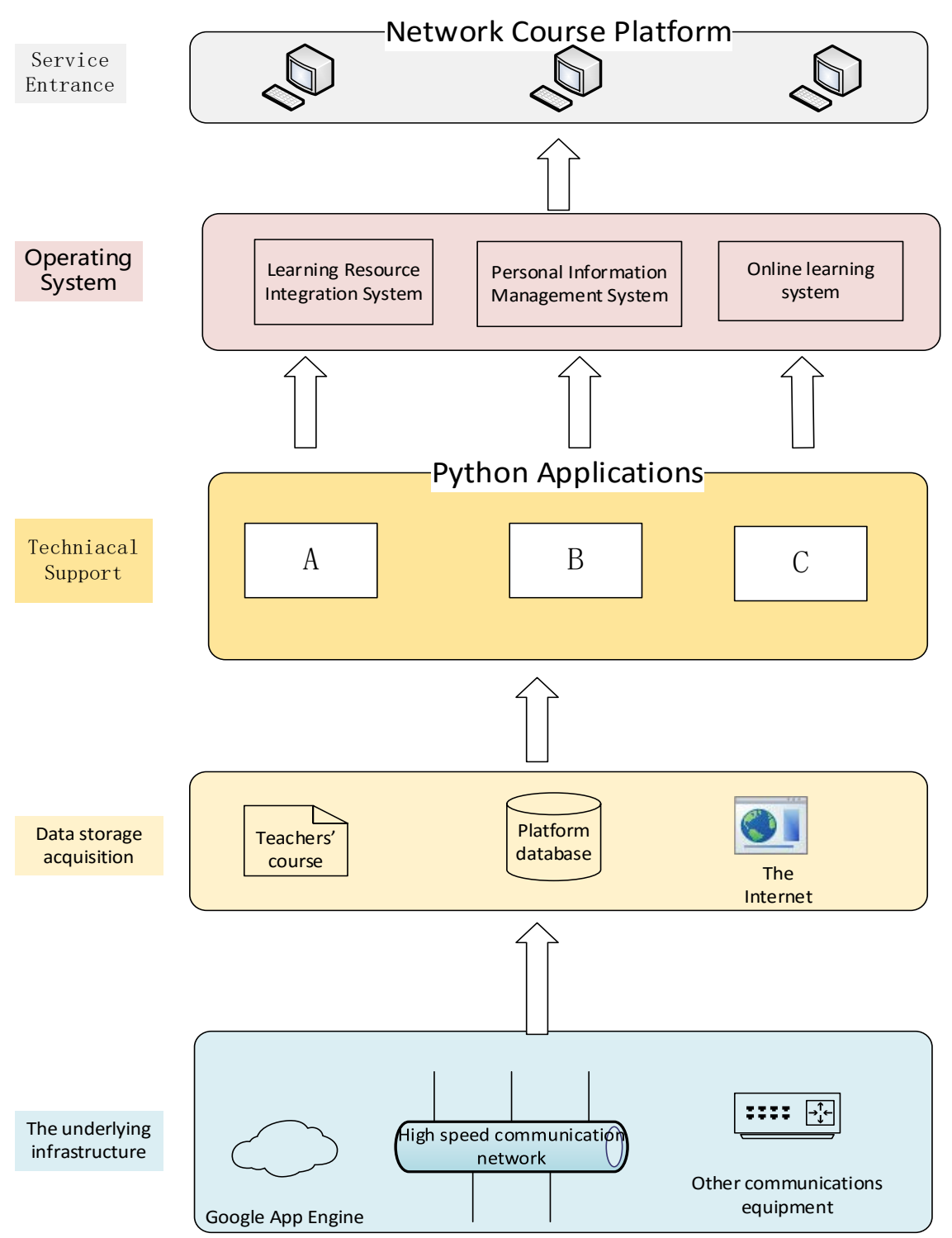

Fig. 3

\section{Conclusion}

Based on previous research, we do needs analysis about web-based courses under cloud computing environment. Then Combining Google and the Google engine application software lay the foundation for the online courses for students. On the basis of the system user guidance, a comprehensive understanding of learning and meticulous research, networking courses will apply advanced educational technology and teaching ideas to practice, detailed analysis, functional definition of the system's performance according to actual needs. We will build a student teacher before class, after-school classes can communicate with the living area of study.

\section{Reference}

[1]Eugene Ciurana, Developing with Google App Engine, First Edition, 2009,1-3

[2] Fosnot C T. Constructivism: Theory, perspectives, and practice. Teachers College Press, 2013

[3]Huang huilin. Movie audience theory. Beijing normal university publishing group, Beijing ,2007. [4]He Peng. Research and development of cloud computing cloud[D] University of Electronic Science and Technology,2011

[5]Li Jing, Research and Practice of task-based collaborative learning environment of cloud computing[D]; Hebei Normal University,2011 